\title{
Pathogenesis, presentation, and treatment of lumbar spinal stenosis associated with coronal or sagittal spinal deformities
}

\author{
Justin F. Fraser, B.A., Russel C. Huang, M.D., Federico P. Girardi, M.D., \\ and Frank P. Cammisa, JR., M.D.
}

Hospital for Special Surgery; Bronx Veterans Affairs Hospital; and the Weill Medical College of Cornell University, New York, New York

\begin{abstract}
Sagittal- or coronal-plane deformity considerably complicates the diagnosis and treatment of lumbar spinal stenosis. Although decompressive laminectomy remains the standard operative treatment for uncomplicated lumbar spinal stenosis, the management of stenosis with concurrent deformity may require osteotomy, laminectomy, and spinal fusion with or without instrumentation. Broadly stated, the surgery-related goals in complex stenosis are neural decompression and a well-balanced sagittal and coronal fusion. Deformities that may present with concurrent stenosis are scoliosis, spondylolisthesis, and flatback deformity. The presentation and management of lumbar spinal stenosis associated with concurrent coronal or sagittal deformities depends on the type and extent of deformity as well as its impact on neural compression. Generally, clinical outcomes in complex stenosis are optimized by decompression combined with spinal fusion. The need for instrumentation is clear in cases of significant scoliosis or flatback deformity but is controversial in spondylolisthesis. With appropriate selection of technique for deformity correction, a surgeon may profoundly improve pain, quality of life, and functional capacity. The decision to undertake surgery entails weighing risk factors such as age, comorbidities, and preoperative functional status against potential benefits of improved neurological function, decreased pain, and reduced risk of disease progression. The purpose of this paper is to review the pathogenesis, presentation, and treatment of lumbar spinal stenosis complicated by scoliosis, spondylolisthesis, or flatback deformity. Specific attention is paid to surgery-related goals, decision making, techniques, and outcomes.
\end{abstract}

\section{Key Words - spinal stenosis • spinal deformity $\bullet$ spondylolisthesis $\bullet$ scoliosis $\bullet$ spinal fusion - lumbar spine}

Lumbar stenosis exists when there is compression of neural elements and their nutrient supply in the central canal, lateral recess, or neural foramen. Stenosis may exist in any or all of these zones concurrently. The prevalence of lumbar spinal stenosis, which should increase as population demographics shift, is reported to be between 1.7 and $8 \%$ in the general population. Lumbar stenosis occurring in a congenitally small canal is termed constitutional stenosis and may present as early as the third decade of life. Most commonly stenosis develops from degenerative changes in the setting of a previously normal spinal canal. Classically, degenerative stenosis presents in the fifth and sixth decades. The current gold-standard treatment for uncomplicated lumbar spinal stenosis is neural element decompressive laminectomy with or without lateral recess and foraminal decompression.

Complex lumbar stenosis presents concurrently with other spinal deformities such as spondylolisthesis, scolio-

Abbreviations used in this paper: $\mathrm{AP}=$ anteroposterior; $\mathrm{CT}=$ computerized tomography; $\mathrm{MR}=$ magnetic resonance; $\mathrm{VB}=$ vertebral body. sis, or lumbar kyphosis (flatback deformity). These lesions may be idiopathic, degenerative, or surgery induced and can complicate presentation and surgical treatment of lumbar spinal stenosis. Additionally, independent rigid sagittal-plane deformities can also complicate spinal stenosis and must be effectively addressed in therapeutic plans. Evaluation and treatment of complex lumbar stenosis requires an understanding of stenosis in the context of these complicated deformities. The pathophysiological features, clinical history and examination, and radiographic findings distinguish the presentation of stenosis that is complicated by spondylolisthesis, scoliosis, or flatback deformity. A thorough understanding of these principles is essential to the appropriate treatment of complex lumbar stenosis.

\section{LUMBAR STENOSIS AND ASSOCIATED DEFORMITIES}

\section{Pathogenesis of Disease}

Degenerative lumbar spinal stenosis results from pathological degeneration of the facet joints, disc herniation, 
hypertrophy and buckling of the ligamentum flavum, and spondylolisthesis. Degenerative changes in the three-joint complex (the intervertebral disc and two facet joints) explain the fluctuation in symptoms with alterations in posture, load, and duration of load. ${ }^{42}$ Lumbar intervertebral disc degeneration represents a cascade of events involving disc herniation, bulging of the disc and ligamentum flavum into the canal, and resulting chronic facet arthrosis, sclerosis, and osteophytic growth. Hypertrophy of the ligamentum flavum is also an important element in the development of spinal stenosis. Lumbar spinal encroachment induces ligamentum flavum hypertrophy, which further aggravates stenosis. Disease of the nerve roots and cord, however, does not typically result directly from compression of the nerves. Rather, the resulting stenosis causes decreased flow of cerebrospinal fluid, which represents approximately $60 \%$ of the nutritional supply to the cauda equina, and increased venous pressure. In such a scenario, any concurrent spinal deformities may critically compromise the nerve roots and cord, as well as exacerbate neurological symptoms of lumbar stenosis.

Spondylolisthesis can be caused by congenital, developmental, traumatic, neoplastic, or degenerative conditions. In degenerative spondylolisthesis, the most common type observed with lumbar stenosis, anterior/ posterior displacement of a VB results from facet joint erosion and attenuation of the muscular, capsular, and ligamentous structures. Fourfold more common in females than in males, degenerative spondylolisthesis occurs most frequently at the L4-5 and L5-S1 levels. ${ }^{9}$ Disc degeneration causes spondylolisthesis with resulting segmental hypermobility, compounded by arthritis in sagittal facet joints. ${ }^{4}$ These facet joints, which support up to one third of the spinal static compression load and axial loads, lose resistance to shear forces in the sagittal plain. ${ }^{17,46}$ The resulting degenerative anterior vertebral subluxation, which is usually no greater than $30 \%$ of the VB width, and vertebral instability can contribute to the neurogenic claudication of spinal stenosis. Isthmic spondylolisthesis results from bilateral defects in the pars interarticularis; a widening of the central canal occurs as the posterior arch of the cephalad vertebrae remains in place while the VB displaces anteriorly. Although this represents a direct widening of the spinal canal, callus formation around the pars defect can induce overall stenosis of the canal. Spondylolisthesis is often associated with back pain, which is significantly influences the surgeon's selection of treatment methods.

Scoliosis may complicate lumbar stenosis. Most cases can be classified as degenerative, idiopathic, or postsurgical. Idiopathic scoliosis is present when degenerative changes occur in cases of preexisting idiopathic scoliosis. Degenerative scoliosis occurs in previously nonscoliotic spines in which disc and facet joint disease cause coronal deformity. Finally, postsurgical scoliosis may result from surgery-induced compromise of posterior elements or the intervertebral disc. With a prevalence of $3 \%$ in the general population, idiopathic scoliosis may contribute significantly to the presentation of lumbar stenosis. ${ }^{36}$ Degenerative scoliosis is characterized by loss of lumbar lordosis, vertebral slippage (either lateral or anterior) at one or more levels, relatively short curves, and moderate amplitudes of scoliotic curvature. ${ }^{38}$ Asymmetrical vertebral collapse and listhesis contribute to the compromise of nerve roots, and may cause back pain and coronal decompensation. Thus, scoliosis complicates both the presenting symptoms as well as the treatment of lumbar stenosis.

Idiopathic and postsurgical flatback syndrome represent another spinal deformity that may complicate presentation and treatment of lumbar spinal stenosis. For the purposes of this review, we consider the more common postsurgical type. Flatback deformity is a relative reduction of normal lumbar lordosis that may produce fixed sagittal imbalance. Because flatback usually develops in the setting of posterior spinal fusions in which distraction instrumentation has been placed, it is commonly accompanied by junctional degeneration, instability, and stenosis. Other causes include prior spinal trauma and systemic rheumatological illnesses such as ankylosing spondylitis. ${ }^{8}$ The resulting sagittal deformity produces increased biomechanical demands on surrounding muscles, ligaments, and intervertebral discs, which may cause back pain and progressive degeneration. Therefore, the treatment of lumbar stenosis in the setting of flatback deformity must address not only the decompression of neural elements but also sagittal alignment and back pain.

A fixed sagittal spinal deformity may result in added back pain, decreased ability to ambulate, and an inability to stand with both the hips and knees extended. Physiological sagittal alignment of the spine includes a thoracic kyphosis of 25 to $55^{\circ}$ with apex at the T6-7 level and a lumbar lordosis of $40-70^{\circ}$ with apex at the L3-4 interspace. Fixed deviation from such a sagittal alignment represents a deformity, and this can occur throughout the spine. In addition, fixed deformities may present concomitantly with spinal stenosis. The pathogenesis of such deformities may include nonstructural and structural causes. Nonstructural causes include psychopathological factors such as depression, neuromuscular disease, improper posture, and irritative lesions such as those in metastatic disease. Structural causes can be degenerative, congenital, compensatory in response to other spinal lesions, secondary to inflammatory diseases, iatrogenic, or idiopathic. Degenerative causes include lumbar stenosis, facet joint arthritis, and degenerative disc disease. Sagittal deformity may represent compensation for thoracolumbar kyphosis greater than $15^{\circ}$, thoracic lordosis, or lumbar kyphosis. Destructive spinal processes include tumors (primary or metastatic), infections (for example, discitis), trauma, and neuropathic pain. Inflammatory diseases can include ankylosing spondylitis, and diffuse idiopathic skeletal hyperostosis (Forestier disease). Iatrogenic causes include postlaminectomy deformity, malaligned fusion, or instrumentation-induced compression and distraction. With such a range of causes, sagittal deformity represents an important potential comorbidity in the presentation and treatment of spinal stenosis.

\section{Clinical Presentation and Diagnostic Evaluation}

The symptoms of lumbar spinal stenosis are typically insidious in onset; they include back pain (95\%), leg pain (71\%), and weakness (33\%). ${ }^{1}$ Lower-extremity neurogenic claudication presents in approximately $94 \%$ of cases 
and is bilateral in $69 \% .{ }^{19}$ Leg pain is typically relieved by recumbency and sitting, and it may be exacerbated by standing. Patients may describe paresthesias and numbness in association with continued walking and may report that downhill walking is more difficult than uphill walking.

In cases of complex lumbar stenosis patients may present with additional characteristic findings. Those with spondylolisthesis often complain of mechanical back pain exacerbated by activity. This pain may be exacerbated by hyperextension, especially in cases of isthmic spondylolisthesis. In cases in which dynamic instability is present, flexion and extension may be painful. In addition, spondylolisthesis can aggravate symptoms of stenosis when the deformity decreases the size of the spinal canal; patients may describe bilateral leg fatigue and aches, as well as greater comfort when sitting or leaning forward. Patients with scoliosis and lumbar stenosis may present with complaints of balance problems related to coronal decompensation. In addition, many patients may experience early fatiguability and muscle pain on the convexity of the curve. In patients with concurrent flatback syndrome feelings of leaning forward or being "bent over" may be present. ${ }^{13}$ They may stumble while walking and have difficulty walking on uneven ground. Back pain due to flatback syndromes is often musculoskeletal, may develop in the cervical, thoracic, lumbar, and sacral regions, and occurs predominantly in muscles under excessive biomechanical strain. When junctional degeneration develops, degenerated segments may also produce back pain. Patients in whom there is additional rigid sagittal deformity may complain of severe back pain in the region of the deformity, fatigue, and a decreased ability to ambulate. Because of postural decompensation, respiratory symptoms and pulmonary problems are common in this patient group.

On physical examination findings in cases of uncomplicated lumbar stenosis range from slight to profound. Tension signs such a straight-leg raises are not typical. A positive Laségue sign (straight-leg raising), however, has been reported in L-5 nerve root compression due to L1-2 stenosis. ${ }^{25}$ Sensory findings may follow dermatomal distributions and are usually present in L-5 (91\%), S-1 (63\%), and L1-4 (28\%) distributions. ${ }^{1}$ A positive response on the "stoop test" may be elicited. This consists of having a patient walk until symptoms develop and then flex the waist; resolution of pain and symptoms is considered positive. ${ }^{12}$ Spinal deformities such as spondylolisthesis, scoliosis, and flatback may alter the ability to perform these tests on physical examination. In addition, the presence of scoliosis and flatback deformity may be evident on direct examination of the spine and posture, with loss of lumbar lordosis, and changes in spinal curvature and compensation (Fig. 1).

Radiographic evaluation of the spine is essential for the diagnosis of complex lumbar spinal stenosis. In uncomplicated stenosis findings on plain radiographs are highly nonspecific, but include short pedicles (developmental stenosis) or degenerative changes in the three-joint complex. ${ }^{42}$ In complex stenosis, plain radiography demonstrates essential information about the magnitude and location of the deformity in sagittal and coronal planes.

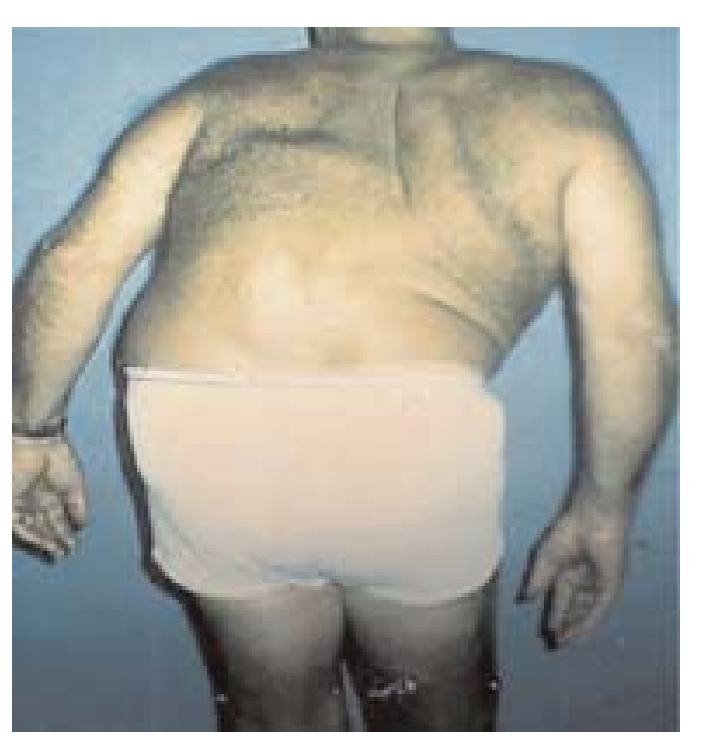

Fig. 1. Photograph obtained during physical examination. The patient complained of low-back pain and leg pain. Scoliotic curvature, loss of lumbar lordosis, and a stance with the leg in the flexed position are demonstrated.

Full-length standing radiography provides information about sagittal and coronal decompensation. Flexion-extension lateral radiography may reveal dynamic instability and provide vital information about the flexibility of flatback deformity.

For noninvasive evaluation of lumbar stenosis, MR imaging is recommended after initial evaluation with plain radiography. Magnetic resonance imaging allows neural compression to be visualized in multiple planes ${ }^{42}$ Furthermore, MR images demonstrate detail that provides insight into the root cause of stenosis. Pathological changes in the intervertebral disc can be visualized. Ligamentum flavum hypertrophy and degeneration may be marked by focal calcification. ${ }^{41}$ Hypertrophic arthritic facet joints may also be identified. Computerized tomography scanning provides excellent visualization of osseous structures and, when combined with myelography, allows superior images of neural compression. In patients in whom MR imaging is precluded for medical or psychiatric reasons, CT scanning is also useful (Figs. 2 and 3).

\section{Principles of Treatment}

The natural history of lumbar spinal stenosis is variable. Johnsson, et al. ${ }^{26}$ followed 32 patients with untreated lumbar spinal stenosis for 4 years and found that $70 \%$ reported unchanged symptoms, whereas $15 \%$ improved and $15 \%$ reported worsened symptoms. In relation to disease progression, they found that patients with a narrower AP canal diameter fared worse, that bilateral symptoms were more likely to persist, and that in $38 \%$ of the cases electromyographic changes were consistent with disease progression. Thus, although many patients with lumbar spinal stenosis may remain clinically stable, a significant number will progress and require treatment for symptomatic disease. Concurrent spinal deformities complicate progression of symptoms, generate additional symptoms such as back pain, and mask the progression of lumbar 


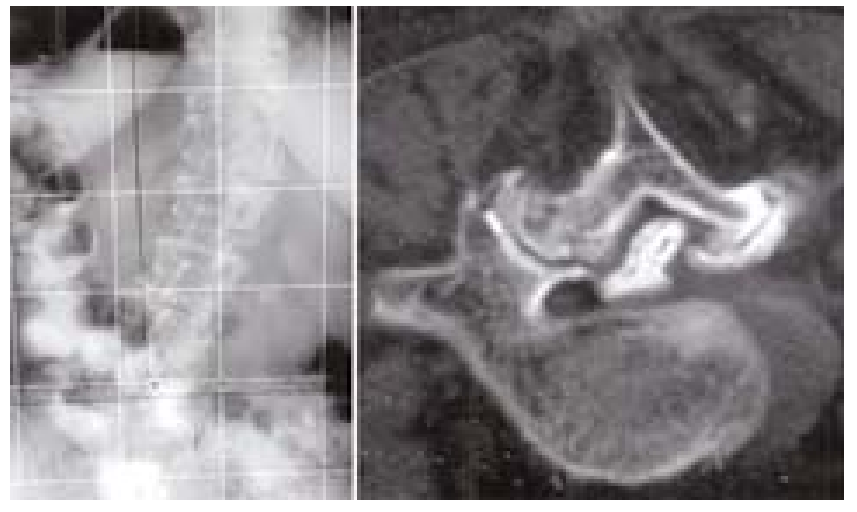

Fig. 2. An AP radiograph (left) and axial CT myelogram (right) demonstrating degenerative scoliosis associated with lumbar spinal stenosis. The CT myelogram reveals the anatomy of the spinal canal.

stenosis. It is important to note that there are no studies in which authors have specifically addressed the natural history of spinal stenosis complicated by spondylolisthesis, scoliosis, or flatback deformity.

Nonoperative treatment for lumbar spinal stenosis consists of lifestyle modifications and pharmacotherapy. Lifestyle modifications include education about the disease as well as movements and activities that exacerbate symptoms; exercise (including modalities such as yoga and tai chi); and physical therapy. Hormone replacement therapy, calcitonin, calcium supplements, and bisphosphonates may help patients with degenerative bone changes and may aid in comorbidities such as osteoporosis. In the medical treatment of symptoms, pain management with nonsteroidal antiinflammatory drugs is the standard. In addition, epidural steroid injections represent an important therapeutic tool for patients with significant surgery-related risk factors or who wish to delay surgery ${ }^{44}$ Some of these risk factors include cardiac disease, hypertension, and respiratory insufficiency. ${ }^{40}$ In addition, epidural steroid injections may provide useful diagnostic and prognostic information. Careful injection technique, however, is important because serious complications have been reported.
Although many cases may be successfully managed nonsurgically, it is clear that many patients derive significant benefit from timely surgical intervention. Amundsen, et al., ${ }^{2}$ reported on 100 patients in whom they compared surgical and conservative treatment for lumbar stenosis. At 4-year follow up, although $50 \%$ of patients in the conservative treatment group (68 cases) experienced good to excellent outcomes (as reported by both physicians and patients), $80 \%$ of those who underwent surgery experienced excellent outcomes. As such, surgery represents an important treatment modality for spinal stenosis.

Indications for surgical intervention in lumbar stenosis include severity of functional limitation and lack of response to conservative treatment. ${ }^{18}$ Intractable pain and/or progressive or significant neurological deficit (for example, foot drop) are the most widely accepted indications for surgery. The ideal surgical candidate is one who suffers significant functional disability, in whom nonsurgical treatment has failed, and has significant correlating disease has been demonstrated on imaging studies. ${ }^{18}$ Other indications include bladder/bowel dysfunction (cauda equina syndrome). In cases of concomitant fixed sagittal deformity, additional indications include a patient's desire for improved appearance and/or increased ambulation, severe back pain, and restoration of impaired visual fields that may result from a forced downward gaze. The principles of surgical treatment in cases of uncomplicated lumbar stenosis include neural element decompression with preservation of facet joints and stability. In cases of complex stenosis, in addition to neural decompression, fusion to achieve intervertebral stabilization as well as realignment of the spinal column may be necessary. To reach these goals, a surgeon may perform a variety of techniques including laminotomy, laminectomy, discectomy, spinal fusion, and osteotomy.

\section{Surgical Treatment}

Laminectomy remains the gold-standard procedure for decompression of lumbar stenosis. Many authors are currently investigating techniques for neural decompression in which there is relative sparing of the integrity of the posterior elements, including laminotomy and lumbar

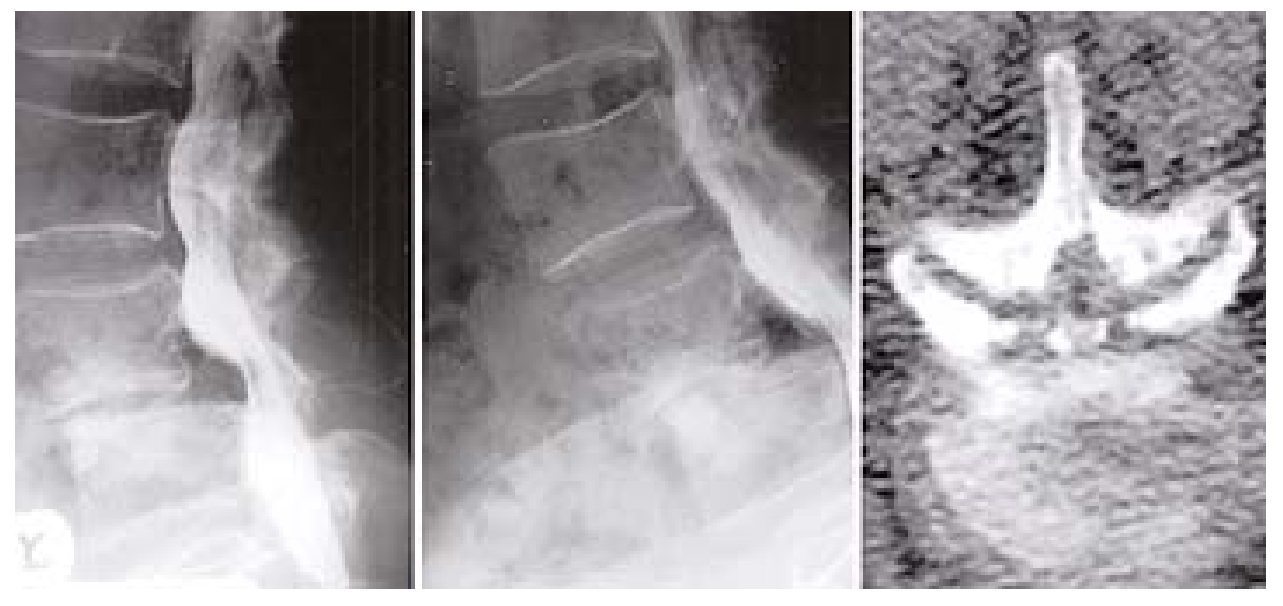

Fig. 3. Myelograms (left and center) and CT myelogram (right) revealing L-5 retrolisthesis with spinal canal narrowing. 
laminoplasty. ${ }^{31}$ The results of decompressive surgery in cases of uncomplicated spinal stenosis can be excellent. In a study of 103 consecutive patients who underwent decompressive surgery for lumbar stenosis, Hansraj, et al. ${ }^{20}$ reported that $94 \%$ were satisfied with their pain relief and only $5 \%$ required a second surgery after a 4-year follow-up period. Excellent results have also been reported after posterior element-sparing procedures such as bilateral laminotomy. ${ }^{39}$ In patients with atypical stenosis and concurrent spinal deformities, however, surgical planning involving decompression becomes more complicated. In addition to neural decompression, other steps must be taken to correct and stabilize the deformity. The emerging consensus in the literature is that patients with spinal stenosis and spondylolisthesis or scoliosis benefit from decompression and fusion and that patients with flatback deformity may require osteotomies and fusion. The placement of instrumentation in cases of spondylolisthesis remains controversial.

\section{Spinal Stenosis and Spondylolisthesis}

The standard decompressive technique in patients with both stenosis and spondylolisthesis includes resection of $50 \%$ of the superior and inferior laminae. Lateral recess decompression is conducted, when necessary, by undercutting of the facet joints. The decompression and the free mobility of neural elements must be verified. In cases in which no flexion-extension instability exists and in which neural compression is confined to the joint level, laminotomy may provide adequate decompression., ${ }^{4,34}$

Although some authors have reported good results after nonfusion-related decompression, the emerging consensus is that decompression and posterolateral fusion offer the best clinical results (Fig. 4). ${ }^{24}$ Herkowitz and Kurz ${ }^{23}$ undertook a prospective randomized trial in which they compared decompression and posterolateral intertransverse fusion with decompression alone in 50 patients in whom degenerative spondylolisthesis was present in conjunction with spinal stenosis; the mean follow-up period was 3 years. Patients who underwent fusion experienced significantly improved clinical outcome. Interestingly, in $36 \%$ of those who underwent fusion radiographic pseudarthrosis developed, but this did not appear to affect clinical outcome at 2 years. Similarly, in a study of 34 elderly patients with lumbar spinal stenosis, Yone, et al., ${ }^{47}$ demonstrated better postoperative outcomes (as measured with the Japanese Orthopedic Association score for low-back pain) in patients who underwent decompression and fusion compared with decompression alone when spinal instability was noted. Finally, although Konno and Kikuchi $^{29}$ did not find significant differences in postoperative VB slippage in 88 patients who underwent either decompression alone (42 cases) or decompression and Graf system stabilization (46 cases), those who underwent stabilization reported significantly lower rates of persistent low-back pain at both 1- and 3-year follow up.

Although the benefits of spinal fusion in cases of spondylolisthesis and stenosis are well recognized, the role of instrumentation remains controversial. Many authors believe that instrumentation offers no clear benefit. Katz, et al. ${ }^{27}$ studied 105 patients with lumbar stenosis and concomitant spondylolisthesis ( $\geq 5 \mathrm{~mm}$ ) and/or scoliosis ( $\geq$ $15^{\circ}$ ), who underwent decompression alone or instrumentation-assisted fusion. Patients in whom noninstrumented fusion was performed experienced significantly greater improvements in back pain severity than those in whom instrumentation was placed. In a study of 130 patients undergoing posterolateral fusion randomized to an instrumentation (64 cases) and noninstrumentation group (66 cases), Christensen, et al., ${ }^{10}$ found that patients with Grades I and II spondylolisthesis experienced superior long-term outcomes (as measured by the Dallas Pain Questionnaire and Low Back Pain Rating Scale) at 5-year follow-up review when treated with noninstrumented fusion. Furthermore, pedicle screw-augmented fusion instrumentation was associated with a higher reoperation rate than noninstrumented fusion. In a prospective study of 77 patients with spondylolisthesis undergoing posterolateral fusion randomized to a transpedicular instrumentation group or an instrumentation group, Möller and Hed-

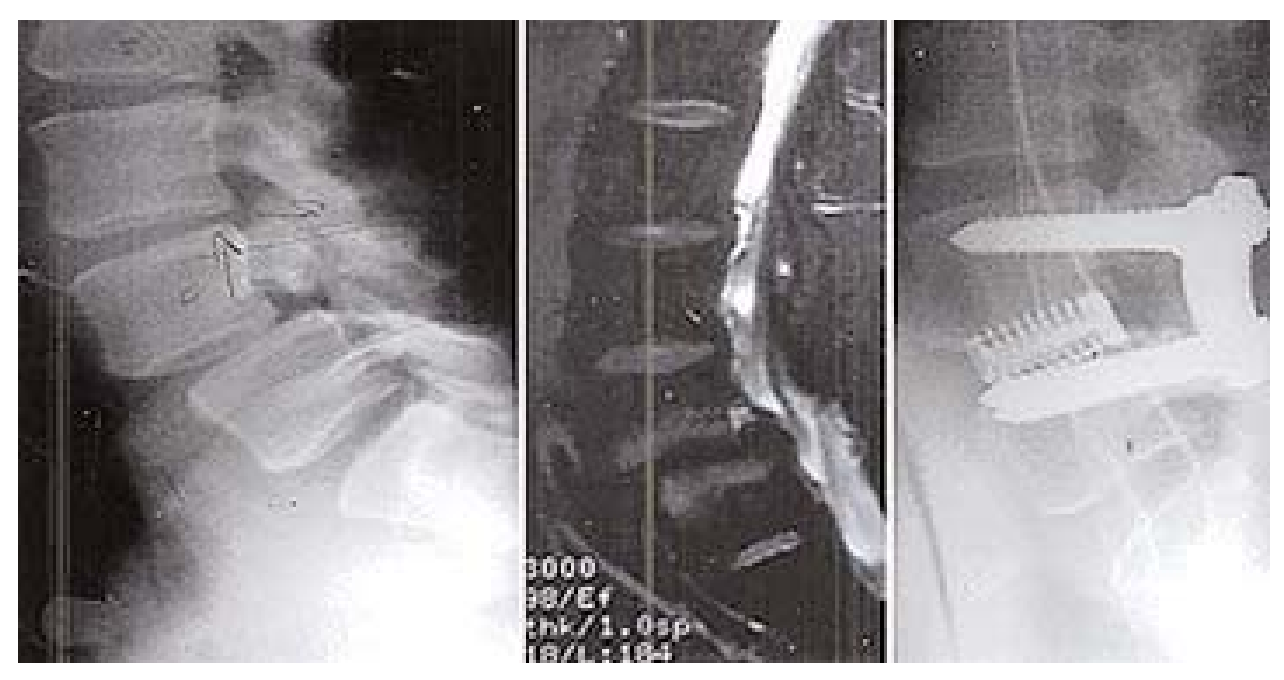

Fig. 4. Left: Lateral radiograph revealing L4-5 spondylolisthesis. Center: Sagittal $\mathrm{T}_{2}$-weighted MR image also demonstrating L4-5 spondylolisthesis. Right: The patient was successfully treated with posterolumbar interbody fusion and instrumentation. 
lund ${ }^{37}$ documented no significant benefit associated with instrumentation. They found that, although instrumentation-assisted fusions entailed significantly longer operative times and significantly greater blood loss, there was no significant difference in fusion rate, Disability Rating Index, or pain index at 2-year follow-up review.

In contrast, Bassewitz and Herkowitz ${ }^{4}$ have proposed that short-term results may be similar regardless of whether instrumentation is used but that instrumentationrelated long-term results are superior. In 1997 Fischgrund, et al. ${ }^{14}$ reported a prospective randomized trial of decompressive lumbar laminectomy and arthrodesis; the patients, all of whom suffered degenerative spondylolisthesis and spinal stenosis, were randomized into an instrumentation-treated or noninstrumentation group. At 2-year follow up, although significantly higher rates of fusion were documented in instrumentation-treated cases $(82 \%$ compared with $45 \%$ in uninstrumentation-treated cases), there was no statistically significant difference in clinical outcome. Fischgrund, et al., claimed that fibrous union (pseudarthrosis) may provide adequate strength to prevent instability and pain at 2-year follow up. In 2000, however, Kornblum, et al., ${ }^{30}$ performed a secondary review of the patients of Fischgrund, et al and Herkowitz, et al., ${ }^{23}$ at 5- to 14-year follow up. They found that clinical results were excellent to good in $86 \%$ of patients in whom radiography demonstrated fusion, whereas only in $56 \%$ of those with a pseudarthrosis. They theorized that fibrous union (pseudarthrosis) may provide stability and good clinical results in short-term follow up but that these results deteriorate over time. Because instrumentation has been shown to increase fusion rates and because successful fusion yields superior long-term results, Bassewitz and Herkowitz ${ }^{4}$ currently recommend the placement of instrumentation in patients undergoing fusion for degenerative spondylolisthesis with stenosis.

Although the standard fusion procedure for spondylolisthesis and stenosis is posterolateral intertransverse fusion, occasionally anterior-posterior fusion may be indicated. Indications for extensive circumferential fusion in spondylolisthesis include patient-related comorbidities known to affect fusion stability (diabetes, smoking, immunocompromise), mechanical factors that make less extensive fusions more predisposed to failure, and postsurgical factors such as previous failed fusion..$^{22}$ Whereas anterior-posterior fusion offers the advantage of higher fusion rates, disadvantages include the greater surgery-related risk, patient morbidity, and potential complications associated with the need for two surgical procedures. ${ }^{22}$

It is important to note that not all patients are good candidates for fusion surgery, which is known to increase operative time, blood loss, and cost. ${ }^{33}$ Many authors have attempted to identify negative prognostic factors in fusion surgery. In a study of 112 patients who underwent revision decompression and instrumentation-assisted fusion for degenerative stenosis, Zheng et al. ${ }^{48}$ found that the number of levels to be fused, a diagnosis of degenerative scoliosis, and excessive body weight had significant effects on the operative time. Length of hospital stay was influenced by advanced age and unemployment associated with three or more comorbid conditions. In a study of 426 patients who underwent lumbar spinal fusion, Andersen, et al., ${ }^{3}$ found that smoking ( $>10$ cigarettes/day) prior to attempted lumbar spinal fusion significantly increased the risk of nonunion (odds ratio 2.01). Finally, Booth, et al., ${ }^{6}$ found that, in 49 patients who underwent decompression, placement of auto/angous bone graft, intertransverse fusion, and implantation of segmental instrumentation, the presence of four or more medical comorbidities was associated with significantly poorer outcome as defined by patient dissatisfaction. In cases of stenosis with concurrent spinal deformity it is important to weigh potential benefits of fusion against potential adverse effects and risk factors for poor outcome.

\section{Spinal Stenosis With Scoliosis}

The surgery-related goals in scoliosis with spinal stenosis are neural decompression, relief of back pain, and restoration of spinal alignment in sagittal and coronal planes. Although prospective data are lacking, the consensus in the literature is that decompression and instrumentation-assisted fusion provide superior clinical outcomes (Fig. 5). Because the scoliotic spine has demonstrated a propensity toward instability, it is intuitive that stabilization is beneficial after a potentially destabilizing decompressive procedure. Hansraj, et al., ${ }^{21}$ reported a series of 50 patients in whom complex lumbar stenosis was defined in previous spinal operations with radiographically documented instability, radiographic evidence of postoperative junctional stenosis, radiographic evidence of instability, degenerative spondylolisthesis greater than Grade I with instability, or degenerative scoliosis with curvature angle greater than $20^{\circ}$; patients underwent decompression and instrumentation-augmented fusion. The authors reported high percentages for satisfaction (96\%), relief of pain $(98 \%)$, satisfaction with ability to walk (94\%), and balance (94\%). In this study scoliosis was not associated with inferior outcomes in terms of back or leg pain. In contrast, Frazier, et al., ${ }^{15}$ prospectively analyzed outcome in 210 patients with stenosis in association with scoliosis or spondylolisthesis. Of the 19 patients with degenerative scoliosis of $15^{\circ}$ or greater, 15 underwent decompression without fusion. Outcomes analysis demonstrated that preoperative scoliosis was associated with less improvement in back pain at 6 and 24 months postoperatively, whereas patients with scoliosis who underwent decompression and instrumentation-assisted fusion did not experience inferior outcomes in the study by Hansraj, et al. ${ }^{21}$

Simmons ${ }^{43}$ classified scoliosis associated with lumbar stenosis into two types: Type I curves, or degenerative curves with minimal or no rotational deformity; and Type II curves, or those that occur when degenerative changes develop in conjunction with a preexisting structural scoliosis with greater rotational deformity. Type II curves are distinguished by greater loss of lordosis, greater length, and clinical history of preexisting scoliosis. Type I curves may be treated with shorter instrumentation and fusions, whereas Type II curves usually require longer instrumentation and fusion as well as sagittal-plane realignment.

Several intraoperative characteristics of the scoliotic spine deserve mention. The dura mater in scoliotic spines is often atrophic and prone to tears. ${ }^{43}$ Many spines with preexisting scoliosis may have been previously treated surgically, in which case the dura may be adherent to the laminae or fusion mass. Furthermore, in spines with sig- 


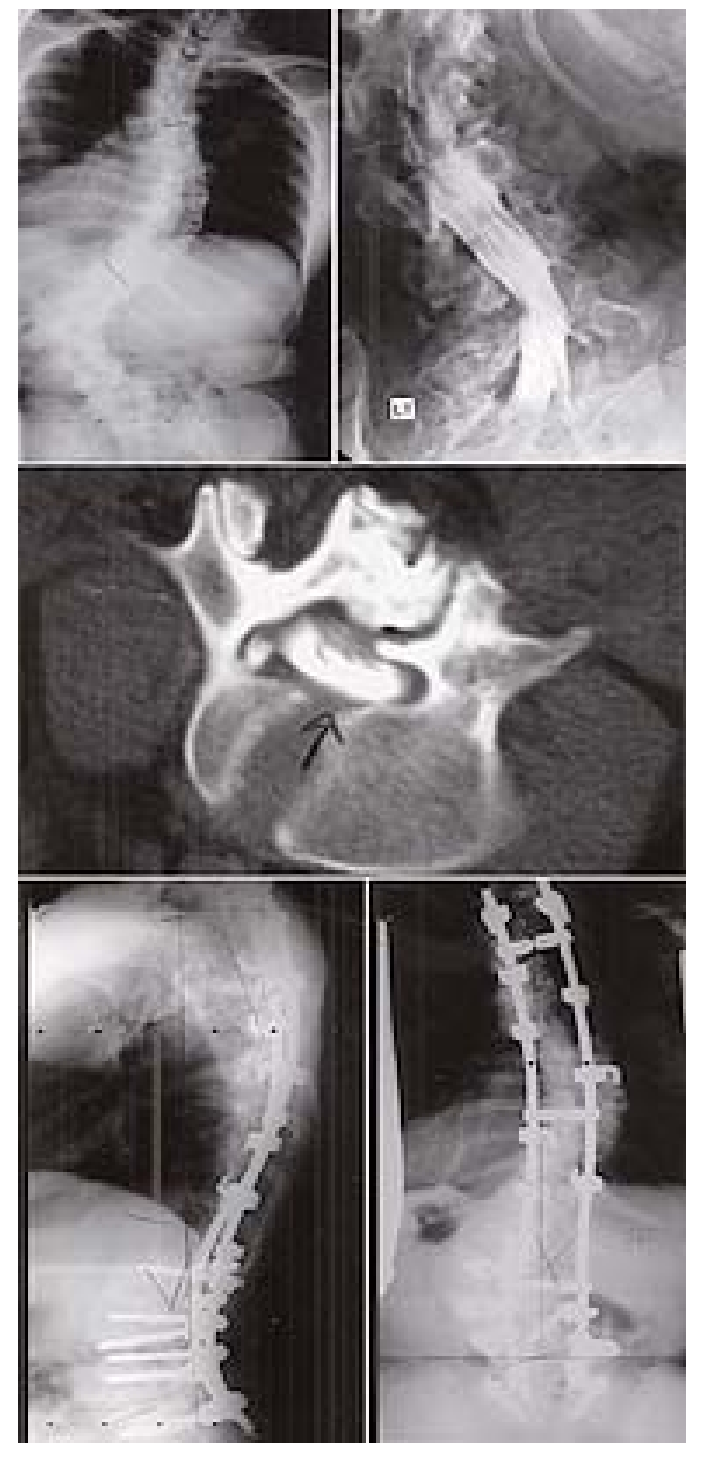

Fig. 5. Imaging studies obtained in a 42-year-old woman with scoliosis, low-back pain, bilateral leg pain, claudication, and evidence of curve progression. A plain AP radiograph (upper left) and myelogram (upper right) demonstrates scoliosis, whereas a CT myelogram (center left) delineates a distorted central canal. The patient underwent anterior (T10-S1) spinal fusion, decompression, posterior thoracic osteotomies, and posterior (T3-S1) spinal fusion. Postoperative AP (center right) and lateral (lower) radiographs demonstrate improvement of spinal balance and extensive correction of deformity.

nificant rotational deformity or postoperative changes, the placement of pedicle screws is challenging. Surgical navigation systems may reduce the incidence of screw misplacement. ${ }^{35}$ In addition, surgical revision in which instrumentation is placed may be necessary in patients with idiopathic scoliosis previously treated with a limited fusion. ${ }^{32}$

Two techniques may be used to obtain correction of scoliotic curves. In Type I curves, short constructs may be placed with concave distraction and a convex neutralization rod; rod contouring to preserve lumbar lordosis is essential. ${ }^{43}$ This construct requires relatively normal pre- operative sagittal alignment. In Type II curves, longer constructs placed in conjunction with rod derotation maneuvers or translational maneuvers in conjunction with sublaminar wires are used. ${ }^{16}$ Again, careful attention must be paid to maintenance or restoration of lumbar lordosis. In all cases, overall spinal balance and sagittal alignment are more important than aggressive correction of the scoliotic curve.

\section{Spinal Stenosis and Flatback Deformity}

Symptomatic flatback deformity commonly presents with a pathological triad of hypolordotic fusion mass, junctional kyphosis, and junctional stenosis defined by Bridwell, et al. ${ }^{8}$ Additionally, areas of pseudarthrosis in the fusion mass may develop localized stenosis. After neural decompression, fusion-related correction of deformity with or without osteotomy is necessary. The flexibility of the deformity is a critical factor in treatment selection. Flexible deformities can usually be treated by anterior-posterior fusion with anterior placement of a structural graft to maintain lordosis, whereas rigid deformities require vertebral osteotomies, decompression, and extension of the fusion mass. ${ }^{8}$

In cases of flatback syndrome involving mechanical back pain but not stenosis, removal of hardware is an option. In a retrospective review, Farcy and $S c h w a b^{13}$ found that partial removal of Harrington rod instrumentation alone in 16 patients, who reported some improvement of back pain after physical therapy, resulted in a temporary decrease in median pain score (at 4 years the score decreased from 4 to 2); however, at a mean 6-year followup examination the score increased to 3 . In this conservatively treated group, the investigators found a mean increase in plumbline from 4.8 to $6.5 \mathrm{~cm}$ at the mean 6-year follow-up examination. In contrast, the study group in which the 16 patients suffered persistent, progressive pain and a falling-forward sensation, required anterior-posterior osteotomy and placement of posterior instrumentation, which resulted in a decrease in mean plumbline from 13.5 to $1.5 \mathrm{~cm}$. Clearly, removal of hardware alone is not an option in patients with concurrent stenosis.

Osteotomy represents the principal tool in the correction of fixed sagittal imbalance in patients with flatback deformity. Techniques for osteotomy range from singlelevel posterior reduction osteotomy to multiple vertebral anterior and posterior osteotomy. In a study of 11 patients with thoracolumbar or lumbar kyphosis, Danisa, et al., ${ }^{11}$ found that single-level posterior reduction (egg-shell) osteotomy decreased the mean kyphotic deformity angle from $-26^{\circ}$ to $+17.5^{\circ}$. Notably, four of 11 patients developed transient postoperative neurological deficits. In a retrospective review of 27 patients who had previously undergone surgery for idiopathic scoliosis, Voos, et al., ${ }^{45}$ studied the effect of anterior-posterior osteotomy at multiple levels on deformity correction and patient satisfaction (as measured by the Scoliosis Research Society outcome questionnaire). Sagittal imbalance was corrected approximately $100 \%$ in all patients, and the mean coronal-plane correction was $68 \%$. They reported nine complications in eight patients (total rate of $30 \%$ of study population) in their 30 patients who underwent multiple osteotomies with segmental instrumentation.

The use of osteotomy and adjunctive fusion techniques 
depends on the nature of stenosis in flatback deformity. In cases of fixed imbalance, Smith-Petersen (shortening of posterior column with lengthening of anterior column) osteotomies or transpedicle decancellation may be used to reduce deformity while improving stenosis. In cases of central stenosis in which Smith-Petersen osteotomies are performed, it is important to decompress the spinal canal and to avoid bone-on-bone junction centrally after closure of osteotomies through a previous pseudarthrosis with placement of an anterior graft. ${ }^{8}$ In contrast, Smith-Petersen osteotomy should not be used at segments where foraminal stenosis is present, because closure may reduce surface area in the foramen. ${ }^{8}$ In general, 10 to $15^{\circ}$ of correction can be obtained at each level when conducting Smith-Petersen osteotomies.

Transpedicle decancellation offers a method of reducing sagittal imbalance in a single segment. This type of osteotomy may be performed in patients with concurrent foraminal stenosis at the target segment, because pedicle resection effectively enlarges the foramen above and below. ${ }^{5}$ In most cases, anterior fusion is required to stabilize the spine unless the prior solidly formed fusion includes the target VB. Approximately 30 to $35^{\circ}$ of correction can be attained per level. Potential complications related to this procedure include dural tear, wound infection, neurological deficit, extensive blood loss, and pseudarthrosis. Although such surgical intervention can yield excellent results, especially in the reduction of pain and deformity, it is important to note that complications may be significant and that major coexistent medical problems (especially four or more) and pseudarthrosis are associated with poor patient satisfaction.?

\section{Spinal Stenosis With Fixed Sagittal Deformity}

In the surgical treatment of spinal stenosis with concomitant fixed sagittal deformity, it is important to treat both pathological processes surgically. In addition to decompression with or without fusion as treatment of the stenosis, one of several other procedures may be necessary depending on the level of the deformity. In cases in which the deformity involves the thoracolumbar region, it can be adequately addressed by posterior decancellation osteotomy; the egg-shell osteotomy procedure may be useful in these cases. ${ }^{5}$ If associated with a scoliotic coronal curve of greater than $40^{\circ}$, however, a combined anterior-posterior fusion may be required. In cases in which the deformity involves the lumbar spine, posterior decancellation or transvertebral osteotomy may be undertaken, with posterior fusion alone or with posterior lumbar interbody fusion/transforaminal lumbar interbody fusion. The posterior/transforaminal lumbar interbody procedures may be especially helpful in cases in which less than $30^{\circ}$ of correction is needed. If an associated scoliotic coronal curve greater than $40^{\circ}$ is present, single-stage anteriorposterior spinal fusion is warranted. Finally, in cases involving the lumbosacral region, posterior decancellation/ transverse decancellation with posterior spinal fusion and prolapsed lumbar intervertebral discectomy (in primary disc cases) are recommended.

\section{CONCLUSIONS}

The surgical treatment of spinal stenosis in association with spondylolisthesis, scoliosis, flatback, or rigid sagittal deformity differs significantly from the treatment of uncomplicated stenosis. In addition to neural decompression, clinical outcomes in cases of complex stenosis are optimized by undertaking fusion, usually in conjunction with placement of instrumentation. Instrumentation-augmented fusion allows for reduction and stabilization of deformity as well as maintenance or reestablishment of coronal and sagittal balance. With appropriate selection of technique for each patient's deformity, a surgeon may profoundly improve pain, quality of life, functional capacity, and cosmesis. The decision to perform surgery entails weighing risk factors such as age, comorbidities, and preoperative functional impairment against potential benefits of improved neurological function, decreased pain, and reduced risk of disease progression. Important predictors of outcome after spinal stenosis surgery include the patient's own assessment of health and health expectations as well as cardiovascular comorbidities. ${ }^{28}$ Ultimately, careful clinical discussion and planning of treatment approach, including conservative and surgical therapies, can result in good outcomes for both the physician and the patient.

Further research is warranted to resolve many questions regarding the natural history of these disorders. It must focus on physiological and biomechanical outcomes after surgical interventions and on surgical techniques that provide adequate reconstruction and decompression via less invasive approaches and when placing hardware. Specifically, the role of instrumentation in spondylolisthesis remains to be demonstrated. Although the treatment of complex spinal deformities involves a myriad of different surgical techniques with variable success and complication rates, advances in the field of spinal surgery have greatly improved the outlook of patients afflicted with lumbar stenosis-related complex deformities of the spine.

\section{References}

1. Amundsen T, Weber H, Lilleas F, et al: Lumbar spinal stenosis. Clinical and radiologic features. Spine 20:1178-1186, 1995

2. Amundsen T, Weber H, Nordal HJ, et al: Lumbar spinal stenosis: conservative or surgical treatment? A prospective 10-year study. Spine 25:1424-1436, 2000

3. Andersen T, Christensen FB, Laursen M, et al: Smoking as a predictor of negative outcome in lumbar spinal fusion Spine 26:2623-2628, 2001

4. Bassewitz H, Herkowitz H: Lumbar stenosis with spondylolisthesis: current concepts of surgical treatment. Clin Orthop 384:54-60, 2001

5. Boachie-Adjei O, Girardi FP, Hall J: Posterior lumbar decancellation osteotomy, Margulies JY, Aebi M, and Farcy JPC (eds): Revision Spine Surgery. St Louis: Mosby, 1999, pp 568-574

6. Booth KC, Bridwell KH, Eisenberg BA, et al: Minimum 5year results of degenerative spondylolisthesis treated with decompression and instrumented posterior fusion. Spine 24: 1721-1727, 1999

7. Booth KC, Bridwell KH, Lenke LG, et al: Complications and predictive factors for the successful treatment of flatback deformity (fixed sagittal imbalance). Spine 24:1712-1720, 1999

8. Bridwell KH, Lenke LG, Lewis SJ: Treatment of spinal stenosis and fixed sagittal imbalance. Clin Orthop 384:35-44, 2001

9. Cauchoix J, Benoist M, Chassaing V: Degenerative spondylolisthesis. Clin Orthop 115:122-129, 1976

10. Christensen FB, Hansen ES, Laursen M, et al: Long-term func- 
tional outcome of pedicle screw instrumentation as a support for posterolateral spinal fusion: randomized clinical study with a 5-year follow-up. Spine 27:1269-1277, 2002

11. Danisa OA, Turner D, Richardson WJ: Surgical correction of lumbar kyphotic deformity: posterior reduction eggshell osteotomy. J Neurosurg (Spine 1) 92:50-56, 2000

12. Dickson RA, Millner PA: The ageing spine, in Newman RJ (ed): Orthogeriatrics. Comprehensive Orthopaedic Care for the Elderly Patient. Oxford: Butterworth-Heinemann, 1992, pp 83-96

13. Farcy JP, Schwab FJ: Management of flatback and related kyphotic decompensation syndromes. Spine 22:2452-2457, 1997

14. Fischgrund JS, Mackay M, Herkowitz HN, et al: 1997 Volvo Award winner in clinical studies. Degenerative lumbar spondylolisthesis with spinal stenosis: a prospective, randomized study comparing decompressive laminectomy and arthrodesis with and without spinal instrumentation. Spine 22:2807-2812, 1997

15. Frazier DD, Lipson SJ, Fossel AH, et al: Associations between spinal deformity and outcomes after decompression for spinal stenosis. Spine 22:2025-2029, 1997

16. Girardi FP, Boachie-Adjei O, Rawlins BA: Safety of sublaminar wires with isola instrumentation for the treatment of idiopathic scoliosis. Spine 25:691-695, 2000

17. Grobler LJ, Robertson PA, Novotny JE, et al: Etiology of spondylolisthesis. Assessment of the role played by lumbar facet joint morphology. Spine 18:80-92, 1993

18. Hall H: Surgery: indications and options. Neurol Clin 17: 113-130, 1999

19. Hall S, Bartleson JD, Onofrio BM, et al: Lumbar spinal stenosis. Clinical features, diagnostic procedures, and results of surgical treatment in 68 patients. Ann Intern Med 103:271-275, 1985

20. Hansraj KK, Cammisa FP Jr, O'Leary PF, et al: Decompressive surgery for typical lumbar spinal stenosis. Clin Orthop 384: 10-17, 2001

21. Hansraj KK, O'Leary PF, Cammisa FP Jr, et al: Decompression, fusion, and instrumentation surgery for complex lumbar spinal stenosis. Clin Orthop 384:18-25, 2001

22. Heary RF, Bono CM: Circumferential fusion for spondylolisthesis in the lumbar spine. Neurosurg Focus 13:Article 3, 2002

23. Herkowitz HN, Kurz LT: Degenerative lumbar spondylolisthesis with spinal stenosis. A prospective study comparing decompression with decompression and intertransverse process arthrodesis. J Bone Joint Surg Am 73:802-808, 1991

24. Herron LD, Trippi AC: L4-5 degenerative spondylolisthesis. The results of treatment by decompressive laminectomy without fusion. Spine 14:534-538, 1989

25. Hidalgo-Ovejero AM, Garcia-Mata S, Martinez-Grande M, et al: L5 root compression caused by degenerative spinal stenosis of the L1-12 and L2-13 spaces. Spine 23:1891-1894, 1998

26. Johnsson KE, Rosén I, Udén A: The natural course of lumbar spinal stenosis. Clin Orthop 279:82-86, 1992

27. Katz JN, Lipson SJ, Lew RA, et al: Lumbar laminectomy alone or with instrumented or noninstrumented arthrodesis in degenerative lumbar spinal stenosis. Patient selection, costs, and surgical outcomes. Spine 22:1123-1131, 1997

28. Katz JN, Stucki G, Lipson SJ, et al: Predictors of surgical outcome in degenerative lumbar spinal stenosis. Spine 24: 2229-2233, 1999

29. Konno S, Kikuchi S: Prospective study of surgical treatment of degenerative spondylolisthesis: comparison between decompression alone and decompression with graf system stabilization. Spine 25:1533-1537, 2000

30. Kornblum MB, Fischgrund JS, Herkowitz HN, et al: American Academy of Orthopaedic Surgeons 67th Annual Meeting.
Scientific Program 2000. Degenerative lumbar spondylolisthesis with spinal stenosis: a prospective long-term study comparing fusion and pseudarthrosis. (www.aaos.org/wordhtml/ anmt2000/sciprog/117.htm) [accessed December 20, 2002]

31. Kostuik JP: Controversies in cauda equina syndrome and lumbar disc herniation, in Wiesel SW, Weinstein JN, Herkowitz H, et al (eds): The Lumbar Spine, ed 2. Philadelphia: WB Saunders, 1996, Vol 1, pp 582-587

32. Kostuik JP, Musha Y: Extension to the sacrum of previous adolescent scoliosis fusions in adult life. Clin Orthop 364:53-60, 1999

33. Kuntz KM, Snider RK, Weinstein JN et al: Cost-effectiveness of fusion with and without instrumentation for patients with degenerative spondylolisthesis and spinal stenosis. Spine 25: 1132-1139, 2000

34. Mohaideen A, Nagarkatti D, Banta JV, et al: Not all rods are Harrington - an overview of spinal instrumentation in scoliosis treatment. Pediatr Radiol 30:110-118, 2000

35. McCulloch JA: Microdecompression and uninstrumented single-level fusion for spinal canal stenosis with degenerative spondylolisthesis. Spine 23:2243-2252, 1998

36. Merloz P, Tonetti J, Pittet L, et al: Pedicle screw placement using image guided techniques. Clin Orthop 354:39-48, 1998

37. Möller H, Hedlund R: Instrumented and noninstrumented posterolateral fusion in adult spondylolisthesis - a prospective randomized study: part 2. Spine 25:1716-1721, 2000

38. Postacchini F: Surgical management of lumbar spinal stenosis. Spine 24:1043-1047, 1999

39. Postacchini F, Cinotti G, Perugia D, et al: The surgical treatment of central lumbar stenosis. Multiple laminotomy compared with total laminectomy. J Bone Joint Surg Br 75: 386-392, 1993

40. Rydevik BL, Cohen DB, Kostuik JP: Spine epidural steroids for patients with lumbar spinal stenosis. Spine 22:2313-2314, 1997

41. Saint-Louis LA: Lumbar spinal stenosis assessment with computed tomography, magnetic resonance imaging, and myelography. Clin Orthop 384:122-136, 2001

42. Schonstrom N, Willen J: Imaging lumbar spinal stenosis. Radiol Clin North Am 39:31-53, 2001

43. Simmons ED: Surgical treatment of patients with lumbar spinal stenosis with associated scoliosis. Clin Orthop 384:45-53, 2001

44. Simotas AC: Nonoperative treatment for lumbar spinal stenosis. Clin Orthop 384:153-161, 2001

45. Voos K, Boachie-Adjei O, Rawlins BA: Multiple vertebral osteotomies in the treatment of rigid adult spine deformities. Spine 26:526-533, 2001

46. Yang KH, King AI: Mechanism of facet load transmission as a hypothesis for low-back pain. Spine 9:557-565, 1984

47. Yone K, Sakou T, Kawauchi Y, et al: Indication of fusion for lumbar spinal stenosis in elderly patients and its significance. Spine 21:242-248, 1996

48. Zheng F, Cammisa FP Jr, Sandhu HS, et al: Factors predicting hospital stay, operative time, blood loss, and transfusion in patients undergoing revision posterior lumbar spine decompression, fusion, and segmental instrumentation. Spine 27: 818-824, 2002

Manuscript received November 15, 2002.

Accepted in final form December 11, 2002.

Address reprint requests to: Federico P. Girardi, M.D., Department of Spinal Surgery, Hospital for Special Surgery, East River Professional Building, 523 East 72nd Street, New York, New York 10021. email: girardif@ hss.edu. 